\section{Non-Differentiable Structure in the Generalized Mandelbrot Set}

\section{J. Peinke, J. Parisi, and B. Röhricht}

Physical Institute (Experimental Physics II),

University of Tübingen

\section{O. E. Rössler}

Institute for Physical and Theoretical Chemistry,

University of Tübingen

\section{W. Metzler}

Department of Mathematics, University of Kassel

Z. Naturforschung. 43a, 287-288 (1988);

received January 26, 1988

The generalized Mandelbrot set, described previously, contains - like the original Mandelbrot set - non-differentiable self-similar fractal structures. An example looking like a vortex structure is presented along with a corresponding generalized Julia set.

Mandelbrot [1] discovered that in the parameter space of the complex logistic map a connected region of a characteristic shape exists. It has since been found again in many other complex-analytic systems and is characterized by its recurrence on all scales. It contains self-similar fractal structures in its boundary.

Previously [2] we looked at the structural change of the Mandelbrot set as a formerly zero parameter is given a non-zero value, so that analyticity is destroyed. We found that smooth segments arise on the boundary (see also [3]). The question remained open whether or not non-differentiable self-similar structures survive.

Numerical evidence is presented that vortex-like structures resembling those seen in the outer regions of the Mandelbrot set, under the name of sea-horses [4], survive, see Figure 1.

The structure was obtained for one particular set of initial conditions. For one point in region of parameter space in question, the corresponding Julia set (boundary between exploding and non-exploding points in the plane of initial conditions [5]) is shown in Figure 2. Very similar pictures - with only small local variations - were obtained over the whole region shown in Figure $1 \mathrm{a}$.

Reprint requests to Dr. Jürgen Parisi, Physikalisches Institut, Lehrstuhl Experimentalphysik II, Universität Tübingen, Morgenstelle 14, D-7400 Tübingen, FRG.
There is a difference between these results and analogous results valid in the analytic case [4]. The privileged initial condition which - only for the quadratic logistic map - exists in the analytic case [6] is gone. Therefore, the pictures obtained in parameter space
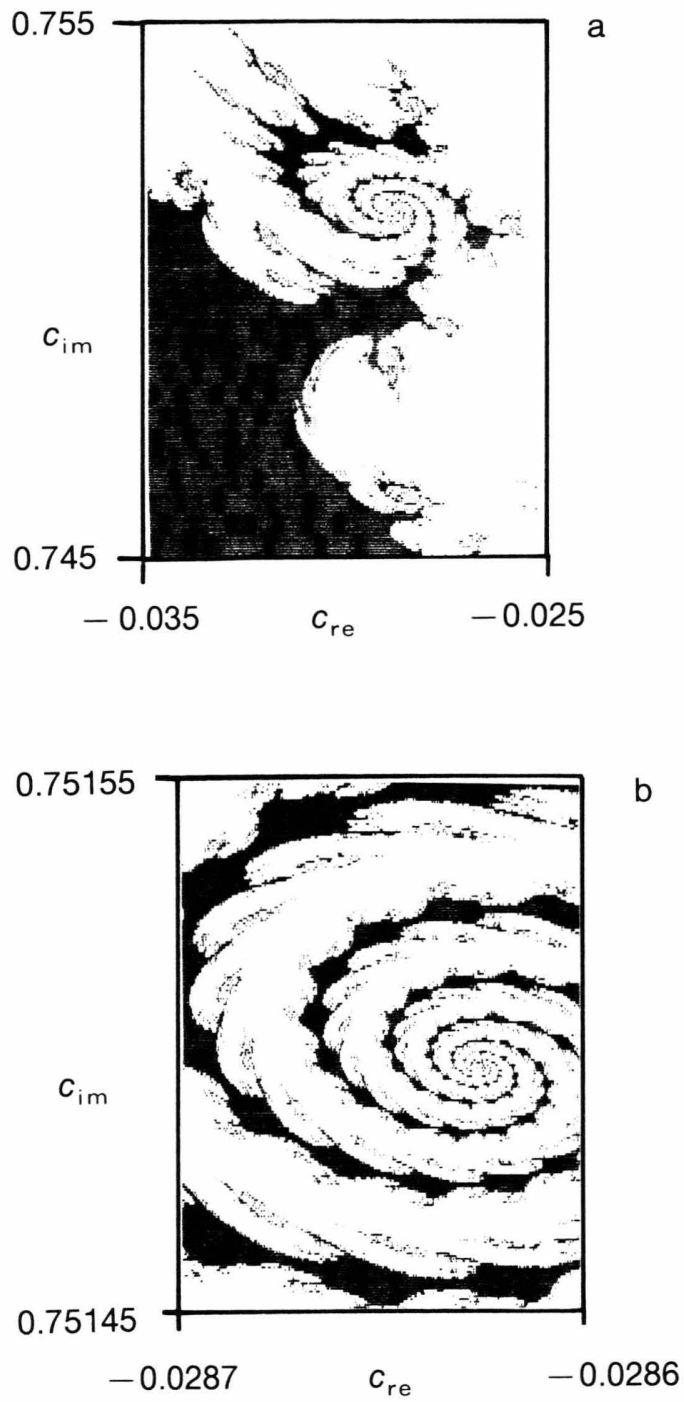

Fig. 1. View of a 2-D slice through the 3-D parameter space of the perturbed complex logistic map, $z_{n+1}=z_{n}^{2}+C$ $+a \operatorname{Re}\left(z_{n}\right)$. Window presented: (a) $c_{\mathrm{re}}:=\operatorname{Re}(C)=-0.035$ to $-0.025, c_{\mathrm{im}}:=\operatorname{Im}(C)=0.745$ to 0.755 ; (b) $c_{\mathrm{re}}=-0.0287$ to $-0.0286, c_{\mathrm{im}}=0.75145$ to 0.75155 . The parameter $a$ is constant $(a=0.3)$. The initial condition was chosen to be $z_{1}=(0.0)$. 


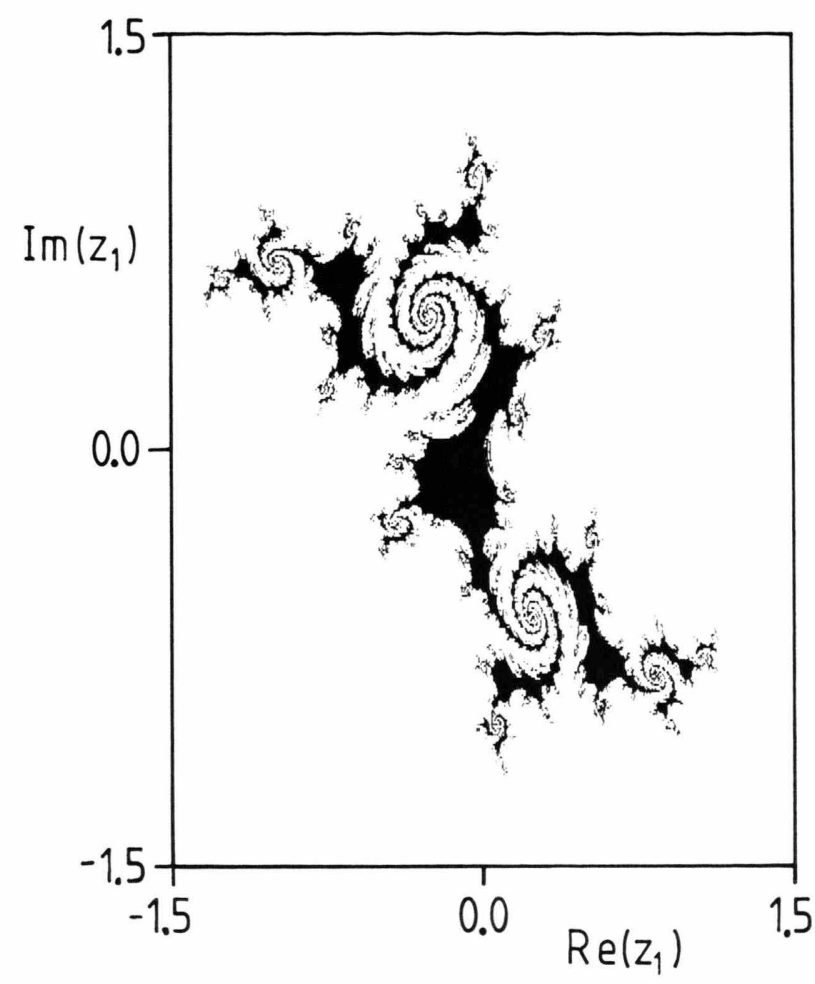

[1] B. B. Mandelbrot, Ann. N. Y. Acad. Sci. 357, 249 (1980).

[2] J. Peinke, J. Parisi, B. Röhricht, and O. E. Rössler, Z. Naturforsch. 42a, 263 (1987).

[3] J. Peinke, J. Parisi, B. Röhricht, O. E. Rössler, and W. Metzler, Z. Naturforsch. 43 a, 14 (1988).

[4] H. O. Peitgen and P. H. Richter, The Beauty of Fractals, Springer, Berlin 1986.
Fig. 2. Julia set-like boundary in the plane of initial conditions, for the parameters $a=0.3, c_{\text {re }}=-0.03, c_{\text {im }}=0.75$. Axes: -1.5 to 1.5 for $\operatorname{Re}(z)$ (abscissa) and -1.5 to 1.5 for $\operatorname{Im}(z)$ (ordinate).

are not invariant with respect to the initial condition chosen.

However, slight variations in the initial conditions result only in slight variations of the shapes encountered. There even is hope that new invariant structures exist in 2-D slices through parameter space which do not depend in their shapes on an optimal or nonoptimal initial condition being chosen. Blanchard [6] considered a similar case for a cubic complex-analytic map. He found an object looking virtually like the original Mandelbrot set. In the present map, there exists a non-differentiable "neck region" (near the point $\left.c_{\mathrm{re}}=-0.20, c_{\mathrm{im}}=0.71\right)$ where again self-similar copies of a whole, generalized Mandelbrot set-like structure occur at all magnifications. The robustness of this object towards changes in the initial conditions is presently being investigated.

This work has partially been supported by the Stiftung Volkswagenwerk.

[5] For analytic maps sometimes called "filled-in Julia set".

[6] P. Blanchard, in Chaotic Dynamics and Fractals, eds. M. F. Barnsley and S. G. Demko, Academic Press, New York 1986, p. 181. 